\title{
Antibacterial Effects and Biocompatibility of Titania Nanotubes with Octenidine Dihydrochloride/Poly(lactic-co-glycolic acid)
}

\author{
Zhiqiang Xu, ${ }^{1}$ Yingzhen Lai, ${ }^{2}$ Dong Wu, ${ }^{3}$ Wenxiu Huang, \\ Sijia Huang, ${ }^{1}$ Lin Zhou, ${ }^{1}$ and Jiang Chen ${ }^{3}$ \\ ${ }^{1}$ School of Stomatology, Fujian Medical University, Fuzhou, Fujian 350000, China \\ ${ }^{2}$ Department of Oral Medicine, Xiamen Medical College, Xiamen, Fujian 361000, China \\ ${ }^{3}$ Department of Oral Implantology, Affiliated Stomatological Hospital of Fujian Medical University, Fuzhou, Fujian 350002, China \\ Correspondence should be addressed to Jiang Chen; dentistjiangchen@sina.com
}

Received 23 January 2015; Revised 11 March 2015; Accepted 13 March 2015

Academic Editor: Zufu Lu

Copyright (c) 2015 Zhiqiang Xu et al. This is an open access article distributed under the Creative Commons Attribution License, which permits unrestricted use, distribution, and reproduction in any medium, provided the original work is properly cited.

\begin{abstract}
Titanium (Ti) implants with long-term antibacterial ability and good biocompatibility are highly desirable materials that can be used to prevent implant-associated infections. In this study, titania nanotubes (TNTs) were synthesized on Ti surfaces through electrochemical anodization. Octenidine dihydrochloride (OCT)/poly(lactic-co-glycolic acid) (PLGA) was infiltrated into TNTs using a simple solvent-casting technique. OCT/PLGA-TNTs demonstrated sustained drug release and maintained the characteristic hollow structures of TNTs. TNTs (200 nm in diameter) alone exhibited slight antibacterial effect and good osteogenic activity but also evidently impaired adhesion and proliferation of bone marrow mesenchymal stem cells (BMSCs). OCT/PLGA-TNTs (100 nm in diameter) supported BMSC adhesion and proliferation and showed good osteogenesis-inducing ability. OCT/PLGA-TNTs also exhibited good long-term antibacterial ability within the observation period of $7 \mathrm{~d}$. The synthesized drug carrier with relatively longterm antibacterial ability and enhanced excellent biocompatibility demonstrated significant potential in bone implant applications.
\end{abstract}

\section{Introduction}

Titanium (Ti) implants are widely used clinically because of their high biocompatibility and good mechanical properties [1]. However, implant-associated infections remain as one of the most serious postoperative complications [2]. The high incidence of implant-associated infections can be mainly attributed to the adhered bacteria that form a biofilm; this biofilm provides the bacteria with high resistance to host defenses and antimicrobial therapies [3, 4]. Conventional systemic drug therapies in bones present limitations such as low efficacy, poor bioavailability, and toxicity [5]. Thus, localized delivery of antimicrobial agents with time-effective handling of infection while potentially eliminating problems associated with systemic administration is highly desirable $[6,7]$. Furthermore, Ti bioactivity is not ideal and thus may lead to the formation of a fibrous capsule around the implant [5]. Fibrous tissues can prevent the contact between host immunity sentinel cells and bacteria [2]. Hence, implant coatings with enhanced osteogenic activity and antibacterial property must be used to prevent infections and elongate the service life of Ti implants.

With the advent of nanotechnology, nanostructured materials play fundamental roles in orthopedic research because bones demonstrate a structural hierarchy at the first level in the nanometer regime [8]. Titania nanotubes (TNTs) fabricated on the Ti surface through electrochemical anodization have received considerable attentions in orthopedic research, because of their high bioactivity to promote bone cell growth and cell differentiation, unlike unanodized Ti [9-12]. Moreover, TNTs with controllable dimensions, high surface-to-volume ratio, and hollow structures have been demonstrated to be superior platforms for local antibiotic delivery applications [13-17].

TNTs, as antibiotic carriers, are challenged with two disadvantages that must be addressed before clinical applications. First, the drug release in proposed drug-delivery systems is directed through diffusion of drug molecules 
from the nanotube structure [5]. The release rate is very fast and short, thus limiting the antimicrobial effects to early-stage peri-implant infections. Although polymers and phospholipids were directly coated on the top of drug-loaded TNTs to extend drug release, TNTs were buried in the coating cap; hence, the potential benefit of TNTs in promoting bone growth was diminished [18-20]. Poly(lactic-co-glycolic acid) (PLGA) has been loaded into TNTs through solvent-casting technique to maintain their characteristic hollow structure and sustain drug release [21]. However, to the best of our knowledge, the biocompatibility of TNTs loaded with PLGA remains unknown. Second, bacteria are highly adaptable in nature, which leads to the evolution of strains resistant to conventional antibiotics [22]. Agents working through unspecific modes of action are required to overcome this resistance such as octenidine dihydrochloride (OCT). OCT, approved as a medicinal substance in several European countries, is an established bispyridine antiseptic with a broad activity and is commonly used as wound antiseptic $[23,24]$. It has attracted increasing attentions because of broad antibacterial spectrum including antibiotic-resistant bacteria, noncytotoxicity at suitable doses, satisfactory stability, and smaller possibility to develop resistant bacteria $[25,26]$.

In this study, we investigated the possibility of using composite PLGA-TNTs as a carrier for sustained OCT delivery. Despite active studies on using localized delivery of antimicrobial agents to prevent implant infections, there have been few reports on using antiseptics [27] and none on using nanotubes to deliver antiseptics. In this paper, for the first time, OCT/PLGA was loaded into TNTS through a simple dip-coating process. Sustained antibacterial ability and biocompatibility were systematically investigated.

\section{Materials and Methods}

2.1. Fabrication of TNTs on Ti. TNTs were fabricated through anodization on a Ti sheet. Ti samples (Alfa-Aesar, Ward Hill, MA, USA; $1 \times 1 \times 0.025 \mathrm{~cm}^{3}, 99.8 \%$ purity) were degreased by sonication in acetone and deionized water for $15 \mathrm{~min}$. The samples were then eroded for $10 \mathrm{~s}$ in $4 \mathrm{wt} \% \mathrm{HF}-5 \mathrm{~mol} / \mathrm{L}$ $\mathrm{HNO}_{3}$, followed by rinsing with deionized water and drying in air. A two-electrode electrochemical cell using a platinum sheet as the counter-electrode was used. Anodization was performed using a mixture of $0.50 \mathrm{wt} \% \mathrm{NH}_{4} \mathrm{~F}+10 \mathrm{vol} \% \mathrm{H}_{2} \mathrm{O}$ in glycerol at $60 \mathrm{~V}$ for $5 \mathrm{~h}$. After anodization, the samples were rinsed with deionized water, dried in air, and annealed at $450^{\circ} \mathrm{C}$ for $2 \mathrm{~h}$.

2.2. OCT Loading. Two types of samples were prepared. The first type was prepared by loading the mixture of OCT/PLGA into TNTs. PLGA (Sigma-Aldrich, St Louis, MO, USA; lactide-to-glycolide ratio: $65: 35,24,000 \mathrm{Da}-$ $38,000 \mathrm{Da})$ and OCT (TCI, Shanghai, China) were dissolved in dichloromethane at $15 \mathrm{mg} / \mathrm{mL}$ and $0.5 \mu \mathrm{g} / \mathrm{mL}$, respectively. The polymer/drug mixture was loaded into TNTs using a dip-coating process at $40^{\circ} \mathrm{C}$ for $2 \mathrm{~d}$ and then dried in air. The second type was produced by directly loading OCT into TNTs. OCT was dissolved in low-surface tension solvent (ethanol) and forced into TNTs using a vacuum-assisted physical adsorption method. In brief, $50 \mu \mathrm{L}$ of $40 \mu \mathrm{g} / \mathrm{mL}$ OCT solution was pipetted onto the nanotube surfaces and allowed to dry under a vacuum desiccator at $20^{\circ} \mathrm{C}$ for $1 \mathrm{~h}$. The loading process was repeated three times.

2.3. Specimen Characterization. Scanning electron microscopy (SEM) (JSM-7500F; JEOL, Tokyo, Japan) was used to characterize the morphology of the prepared TNTs and OCT/PLGA-TNTs. The infiltration of PLGA polymer into TNTs was also assessed by SEM according to the report of Jia and kerr [21]. Scotch tape was used to separate the OCT/PLGA-TNTs from Ti foil. The tape with OCT/PLGATNTs then was soaked in 5 vol\% HF solution for 15 min until all TNTs were etched off. The remaining PLGA was examined through SEM.

2.4. Contact Angle Determination. A $1 \mu \mathrm{L}$ drop of distilled water was delivered on the clean specimen surface with a syringe at a room temperature and in the open atmosphere of the lab. Contact angles were measured on the obtained photographs (Phoenix 300; SEO, Seoul, Korea). The mean value was calculated from five separate measurements.

2.5. Protein Adsorption Assay. A $1 \mathrm{~mL}$ droplet of DMEM-LG containing $10 \%$ FCS was pipetted onto each specimen. After incubation at $37^{\circ} \mathrm{C}$ for $2 \mathrm{~h}$, the proteins adsorbed onto the samples were detached using $1 \%$ sodium dodecyl sulfate. The protein concentrations were determined using a MicroBCA protein assay kit (Pierce, Rockford, IL, USA).

2.6. Release Profile of OCT. OCT has been shown to be stable in chloroform at $40^{\circ} \mathrm{C}$ [25]. So, in vitro OCT release kinetics of the samples were measured using ultraviolet visible spectroscopy (UV-1750; Shimadzu, Kyoto, Japan) by recording the absorption peak at $280 \mathrm{~nm}$, which is the characteristic excitation wavelength of OCT. Two specimens, TNTs loaded with OCT and TNTs loaded with OCT/PLGA, were immersed in 1-mL of PBS in a glass vial while rotating (60 revolutions per minute) at $37^{\circ} \mathrm{C}$. After $1,2,4$, and $6 \mathrm{~h}$, as well as after $1,2,3,6,9,12,15$, and $18 \mathrm{~d}, 500 \mathrm{~mL}$ of the solution was sampled and fresh PBS was replenished. Three samples were tested in each time interval, and the mean value was used in data analysis.

2.7. Bacteria Cultures. Staphylococcus aureus (S. aureus) (ATCC25923; American Type Culture Collection, Manassas, VA, USA) was cultivated in the brain-heart infusion broth medium at $37^{\circ} \mathrm{C}$ for $12 \mathrm{~h}$ and then adjusted to a concentration of $10^{6} \mathrm{CFU} / \mathrm{mL}$. The specimens were placed on 24 -well culture plates and separately incubated in $1 \mathrm{~mL}$ of the bacteriacontaining medium.

2.7.1. SEM Observation. After being incubated in $1 \mathrm{~mL}$ of the bacteria-containing medium for $6 \mathrm{~h}$, the samples were rinsed with PBS, fixed with 3\% glutaraldehyde, dehydrated in graded ethanol series, freeze-dried, sputter-coated with thin platinum layers, and observed by SEM. 
TABLE 1: Primers used for qRT-PCR.

\begin{tabular}{lcc}
\hline Gene & Forward primer sequence $\left(5^{\prime}-3^{\prime}\right)$ & Reverse primer sequence $\left(5^{\prime}-3^{\prime}\right)$ \\
\hline RUNX2 & CCTCTGACTTCTGCCTCTGG & GATGAAATGCCTGGGAACTG \\
ALP & GCCTGGACCTCATCAGCATT & AGGGAAGGGTCAGTCAGGTT \\
OCN & CAAGTCCCACACAGCAACTC & CCAGGTCAGAGAGGCAGAAT \\
COL-I & ATCTCCTGGTGCTGATGGAC & GCCTCTTTCTCCTCTCTGACC \\
GAPDH & GGCACAGTCAAGGCTGAGAATG & ATGGTGGTGAAGACGCCAGTA \\
\hline
\end{tabular}

2.7.2. Antibacterial Assay. In vitro antibacterial activity was assessed by the plate-counting method. After culturing for 1 , 4 , and 7 days, the sample was rinsed in PBS and ultrasonically agitated to detach the bacteria from the sample. The bacteria suspensions were recultivated on agar plates for colony counting. The antibacterial rates were calculated using the following formula: antibacterial rate $(\%)=(\mathrm{CFU}$ of control CFU of experimental groups)/CFU of control $\times 100 \%$, where Ti served as the control while TNTs and TNTs/PLGA constituted the experimental groups.

2.8. Cell Cultures. Sprague-Dawley rat bone marrow mesenchymal stem cells (BMSCs) were purchased from Cyagen Biosciences (Guangzhou, China). The cells were cultured in DMEM-LG containing $10 \%$ FCS at $37^{\circ} \mathrm{C}$, and the medium was changed every $3 \mathrm{~d}$. Cells were used between passage 4 and passage 6 in the following experiments. The samples were placed in 24-well plates, and the BMSCs were seeded at a density of $4 \times 10^{4} /$ well for the cell adhesion assay and $2 \times$ $10^{4}$ /well for the other assays.

2.8.1. Cell Morphology. After culturing for $2 \mathrm{~d}$, the samples were rinsed with PBS, fixed with $2.5 \%$ glutaraldehyde, dehydrated in graded ethanol series, freeze-dried, sputter-coated with thin platinum layers, and observed by SEM.

2.8.2. Adhesion and Proliferation. For the cell adhesion assay, the adherent cells were fixed and stained with 4,6-diamidino2-phenylindole (DAPI; Sigma-Aldrich) after culturing for 0.5, 1 , and $2 \mathrm{~h}$. Images were captured from five random fields by a fluorescence microscope, and the cell number in each field was determined. To assess cell proliferation, the cell numbers were assessed using Cell Counting Kit-8 (CCK-8; Beyotime, Shanghai, China) assay after seeding for 1,3 , and $7 \mathrm{~d}$.

2.8.3. Gene Expressions. The expression levels of osteogenesis-related genes, including runt-related transcription factor 2 (RUNX2, a key transcript factor for osteogenic differentiation), alkaline phosphatase (ALP, an early marker for osteogenic differentiation), osteocalcin ( $\mathrm{OCN}$, a late marker for osteogenic differentiation), and type 1 collagen (COL1 , a main collagen found in bones), were measured using quantitative reverse transcription polymerase chain reaction (qRT-PCR). Total RNA was extracted using Trizol (Invitrogen, Carlsbad, CA, USA) after culturing for 2 weeks. Total RNA was then reverse-transcribed with a cDNA Reverse Transcription Kit (TaKaRa, Shiga, Japan), and qRT-PCR analysis was performed on an ABI Prism 7500 real-time PCR cycler (Applied Biosystems, Carlsbad, CA, USA) using SYBR Premix Ex Taq II (TaKaRa). The primers for the target genes are listed in Table 1. The expression levels of the target genes were normalized to that of the housekeeping gene GAPDH.

2.9. Statistical Analysis. All data were expressed as the mean \pm standard deviation (SD). One-way ANOVA and StudentNewman-Keuls post hoc test were used to determine the level of significance. $P<0.05$ was considered to be significant, and $P<0.01$ was considered to be highly significant.

\section{Results}

3.1. Specimen Characterization. The SEM images of TNTs with and without OCT/PLGA loading are shown in Figure 1. TNTs (200 nm in diameter) were neatly and uniformly arranged over the anodized Ti surface in Figure 1(a). The diameter decreased to $100 \mathrm{~nm}$ after OCT/PLGA loading, and the intertubular areas were filled with PLGA, as shown in Figure 1(b). The side view of TNTs and the image of the remaining PLGA after TNTs removal are shown in Figures 1(c) and 1(d), respectively. The remaining PLGA was uniformly arranged in most of the areas, and its length was similar to the length of TNTs. Thus, PLGA demonstrated an excellent infiltration depth into TNTs.

3.2. Static Contact Angles. Drop images were captured by a video camera in the direction perpendicular to the surface (Figure 2). For hydrophilic material, the contact angle is lower than 90 degrees, and the smaller the contact angle, the greater the hydrophilicity. Multiple comparisons (Figure 2) revealed that TNTs showed the greatest surface hydrophilicity, whereas Ti exhibited the greatest surface hydrophobicity.

3.3. Protein Adsorption Assay. The amounts of adsorbed proteins from $10 \%$ FCS after $2 \mathrm{~h}$ of incubation are presented in Figure 3 to elucidate subsequent cellular responses. OCT/PLGA-TNTs with higher hydrophilicity absorbed more proteins than control Ti. However, paradoxically, TNTs with the highest hydrophilicity had the fewest protein aggregates. It is because the protein aggregates $(\approx 30$-nm-size regime) initially attach only to the available surfaces that are the top portion of the nanotube walls $[11,12]$, and these aggregates are too small to anchor on TNTs with a diameter of $200 \mathrm{~nm}$. The protein aggregates could easily attach to OCT/PLGA-TNTs, probably because the larger intertubular areas, which were filled with PLGA, provided more effective nucleation sites for protein adsorption. 


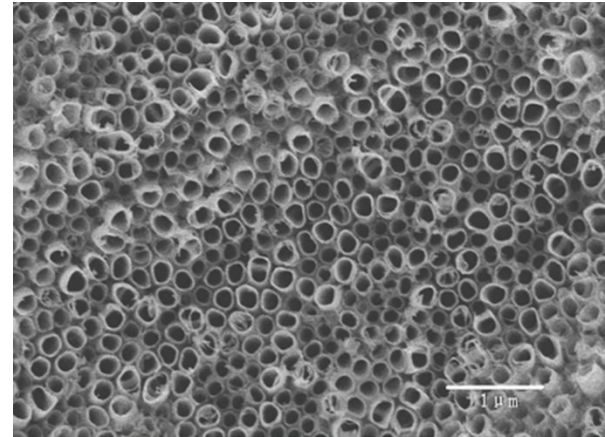

(a)

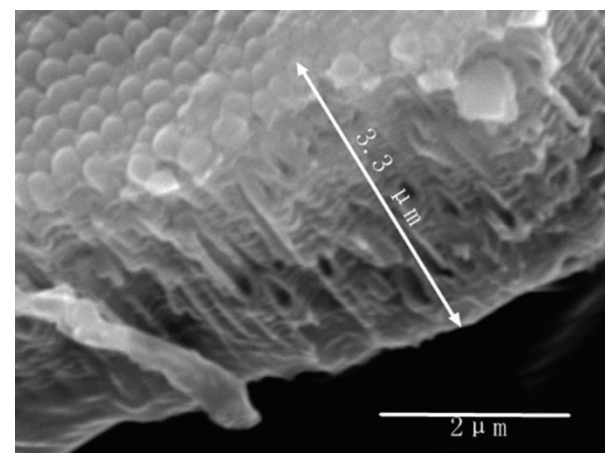

(c)

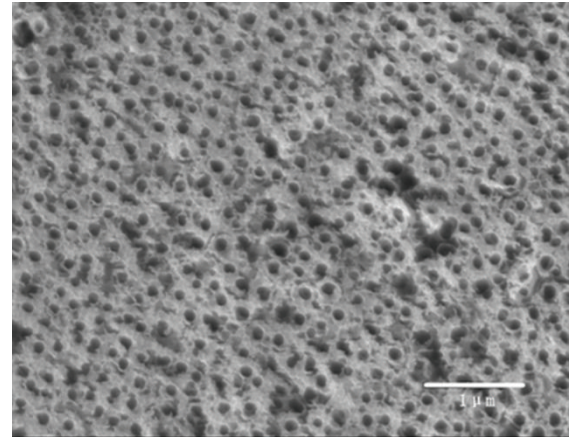

(b)

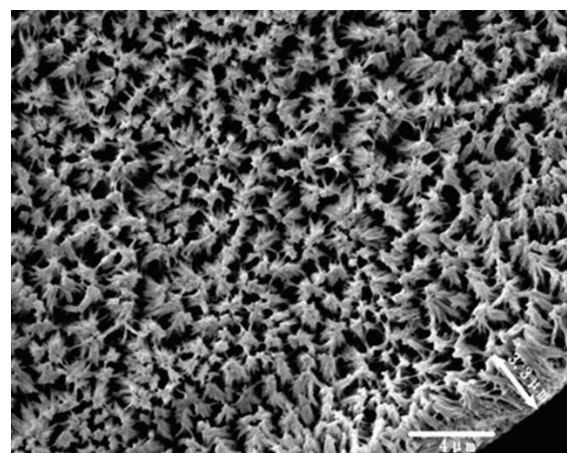

(d)

FIGURE 1: SEM images: (a) TNTs with a diameter of $200 \mathrm{~nm}$, (b) OCT/PLGA-TNTs with a diameter of $100 \mathrm{~nm}$, (c) side view of TNTs, and (d) remaining PLGA.
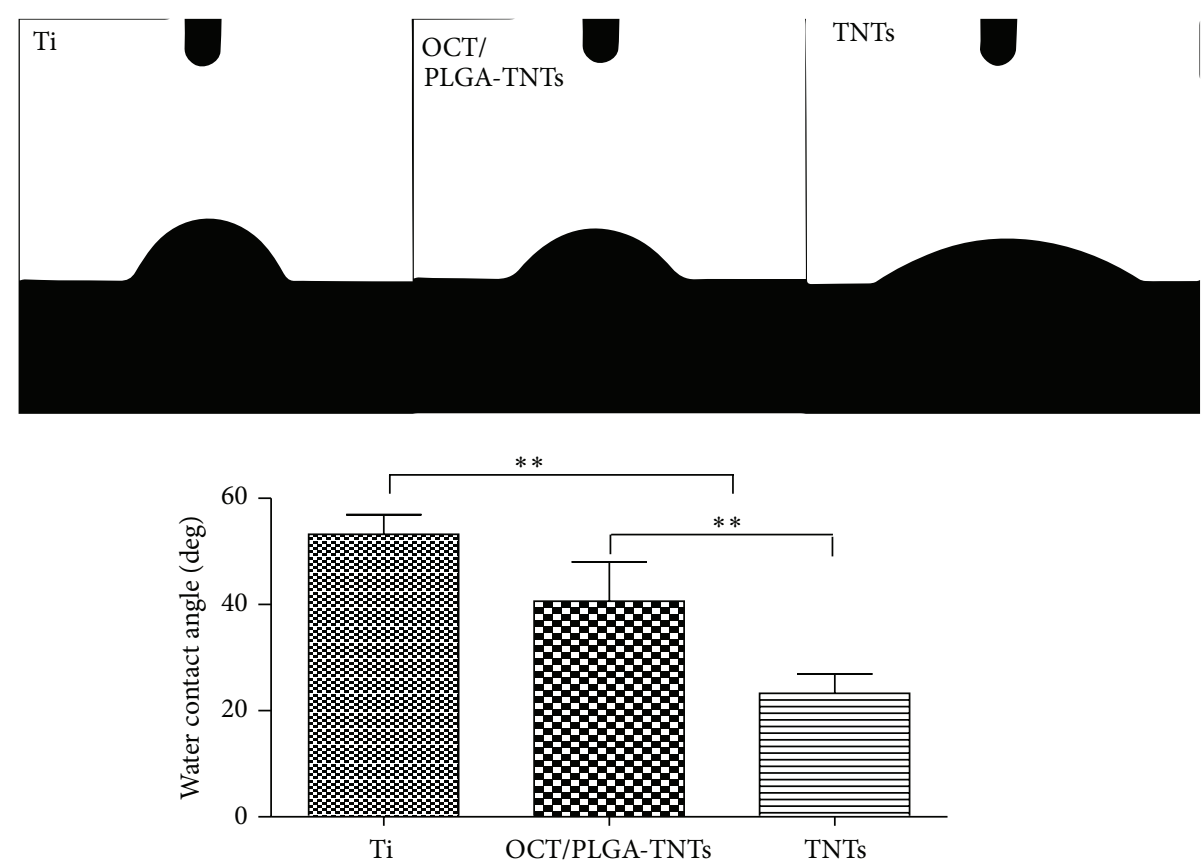

FIGURE 2: Photographs of water droplets on different samples and multiple-comparison results of contact angles on different samples (mean $\left.\pm \mathrm{SD}, N=5,{ }^{*} P<0.05,{ }^{* *} P<0.01\right)$. 


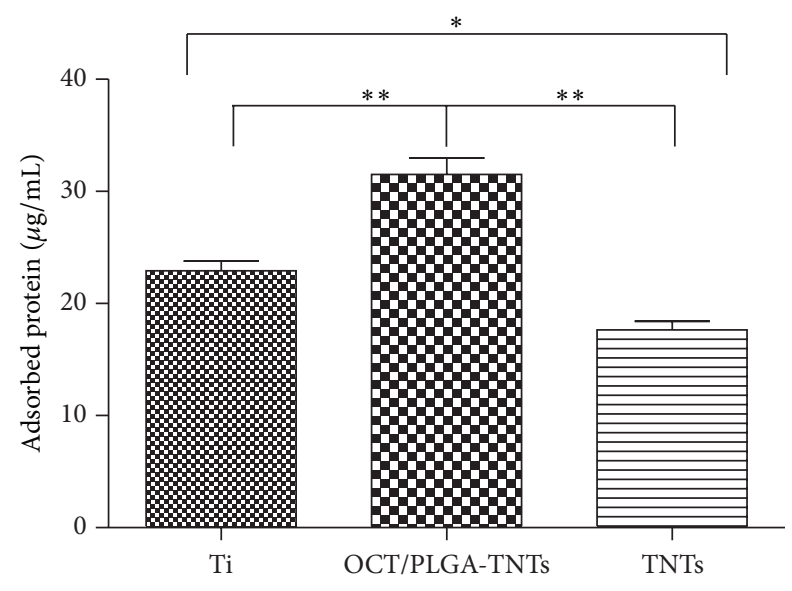

FIGURE 3: Protein adsorption to the samples after $2 \mathrm{~h}$ of immersion in DMEM-LG containing $10 \%$ FCS (mean $\pm \mathrm{SD}, N=3,{ }^{*} P<0.05$, $\left.{ }^{* *} P<0.01\right)$.

3.4. OCT Release. Figure 4 shows the OCT release time profiles from TNTs and OCT/PLGA-TNTs in PBS. For the OCT directly released from the nanotubes, the release kinetics could be described in two phases: a high percentage (80\%) of the drug released in the first $6 \mathrm{~h}$ (initial burst release) and then a slow release for the following $2 \mathrm{~d}$. By contrast, in the case of PLGA-TNTs, the drug release pattern was directed through the transport of drug through the polymer matrix and the rate of polymer degradation $[19,21]$, so the burst release decreased from $80 \%$ to $48 \%$ (versus TNTs) and the extended overall release increased from $2 \mathrm{~d}$ to $15 \mathrm{~d}$.

3.5. Antimicrobial Activity. Figures 5(a), 5(b), and 5(c) show the qualitative SEM assessments of bacteria incubated with samples. It could be observed that many multiple bacterial colonies formed colony masses on the surfaces of $\mathrm{Ti}$ and TNTs. In strong contrast, very few single bacterial colonies were detected on OCT/PLGA-TNTS.

Antibacterial activity was evaluated for $7 \mathrm{~d}$ as shown in Figure 5. On day 1, OCT/PLGA-TNTs loaded with OCT generated a high antibacterial rate of $100 \%$. Although a slight decrease in the antibacterial rates was observed as time increased, a high antibacterial rate of $97.2 \%$ was maintained until day 7. This finding suggests an effective and long-term antibacterial activity against $S$. aureus. TNTs alone exhibited slight antibacterial rate of about $20 \%$, which was relatively constant with time.

3.6. Cell Morphology. The BMSCs displayed dramatically different shapes related to the topography of the substrate, as shown in Figure 6. Most of the BMSCs on Ti appeared round and spread poorly with no cellular extensions and filopodia propagation indicative of undifferentiated BMSCs (Figures 6(a), 6(b), and 6(c)). By contrast, the cells on OCT/PLGA-TNTs (Figures 6(d), 6(e), and 6(f)) and TNTs (Figures $6(\mathrm{~g}), 6(\mathrm{~h})$, and 6(i)) had to extend across the tubes to locate a protein-deposited surface on intertubular areas for initial contact, thereby becoming more extended with a large number of prominent filopodia and unidirectional lamellipodia extensions compared with $\mathrm{Ti}$, and pure TNTs with a larger diameter presented stronger induction.

3.7. Adhesion and Proliferation. The highest numbers of initial adherent cells on OCT/PLGA-TNTs and lowest numbers of initial adherent cells on TNTs are shown in Figure 7. Upon contact of an implant surface with blood, the proteins available in the serum adsorb to the surface within an initial incubation time and mediate the subsequent cellular performance $[11,28]$. Cell attachment is believed to be significantly greater on material surfaces with more protein adhesions [29-33]. Thus, OCT/PLGA-TNTs with more protein adhesions induced more cell attachment at an early time than $\mathrm{Ti}$, and TNTs with the fewest protein aggregates induced the least attachment.

Cell proliferation was measured by the CCK- 8 assay (Figure 8). On day 1, the cell numbers on OCT/PLGA-TNTs were slightly smaller than those on Ti. This slight proliferation suppressive effect was possibly related to the differentiation tendency of BMSCs because of the reciprocal relationship between cell proliferation and differentiation [34, 35]. The inhibition of BMSC proliferation was not serious or long. By days 3 and 7, the cell growth on $100 \mathrm{~nm}$ OCT/PLGA-TNTs caught up because of the large surface area available for cell colonization. This finding indicates that OCT/PLGA-TNTs did not impair cell viability and could support cell proliferation. However, cell proliferation on TNTs was obviously lower than that on the Ti control and OCT/PLGA-TNTs, and this trend became more evident with time. The very low adhesion at the early stage can potentially lead to cell quiescence or even apoptosis by anoikis, a type of programmed cell death through "homelessness" [36].

3.8. Gene Expressions. The expression levels of osteogenesisrelated genes including RUNX2, ALP, OCN, and COL-1 were assessed by qRT-PCR. The results are shown in Figure 9. The three topographies explored in this study induced different gene expression levels. Previous study reported that elongated BMSCs are prone to undergo osteogenesis [37, 38], so the effects of nanotubes on inducing fast and good distribution of BMSCs favored the osteogenic ability. OCT/PLGA-TNTs and TNTs significantly promoted the expression of osteogenesisrelated genes and demonstrated excellent osteogenic activity, with the latter exhibiting a higher promotion.

\section{Discussions}

With the steadily increasing demand for implants, a proper approach that can endow biomaterials with long-term antibacterial ability and biointegration has been actively pursued [28]. In the present study, OCT/PLGA was loaded into TNTs using a simple solvent-casting technique. The design sustained OCT release and maintained the characteristic hollow structure of TNTs. OCT/PLGA-TNTs showed good antibacterial characteristics and biointegration and are thus important to prevent implant-associated infections.

PLGA, which was approved by the US FDA as therapeutic material, was selected as the OCT carrier in this study 


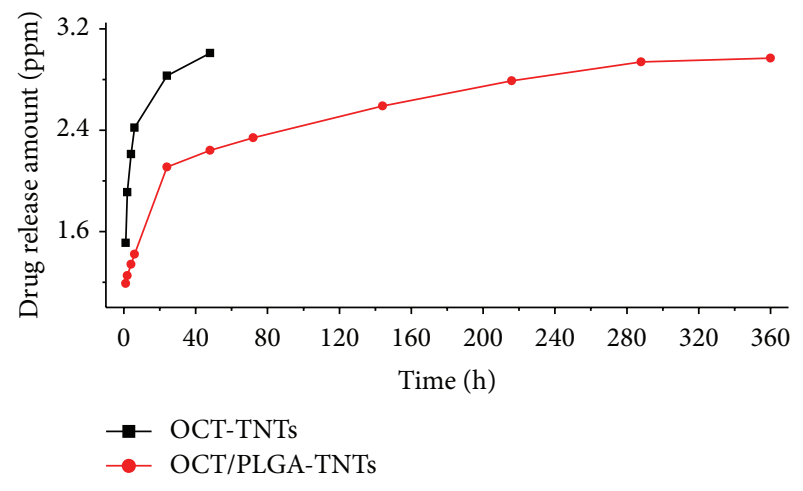

(a)

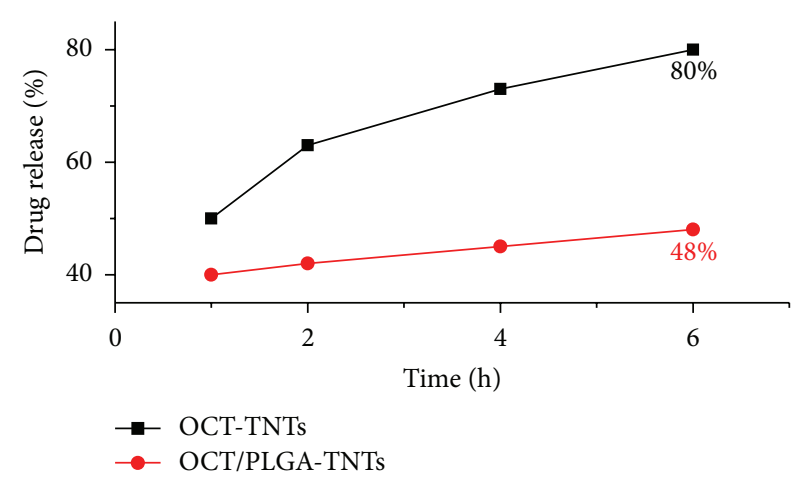

(b)

FIGURE 4: In vitro release profiles in PBS: (a) accumulative amount of OCT release from the samples and (b) accumulative percentage of OCT release from the samples.

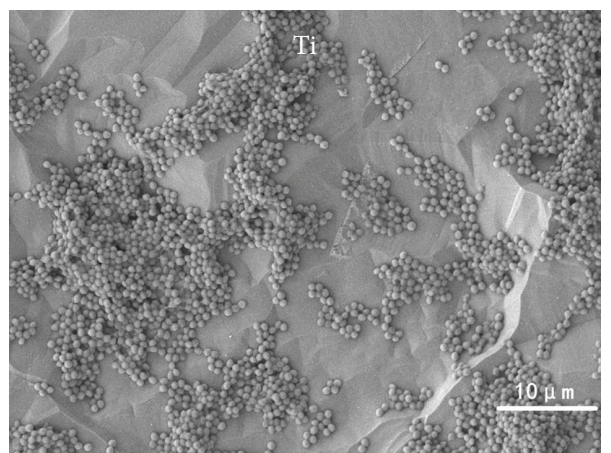

(a)

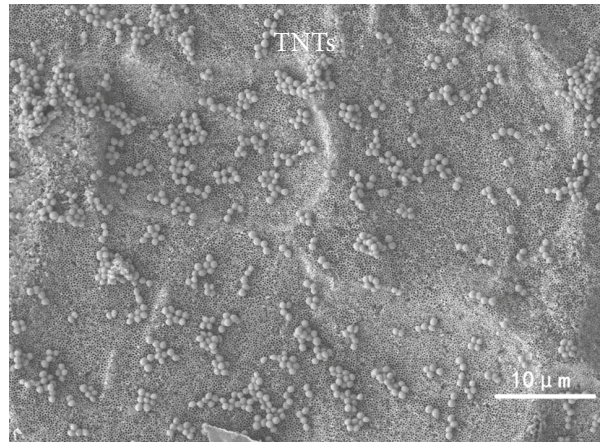

(c)

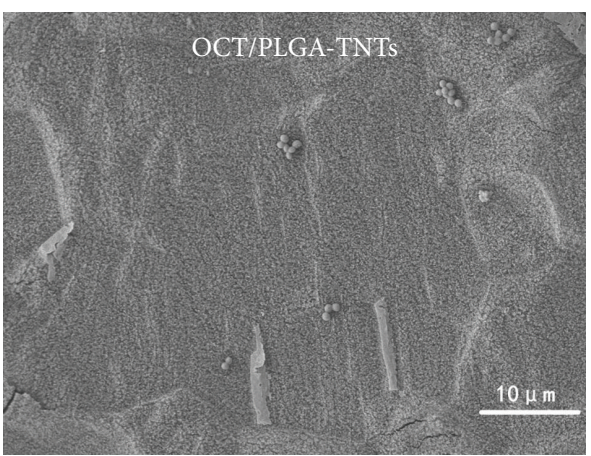

(b)

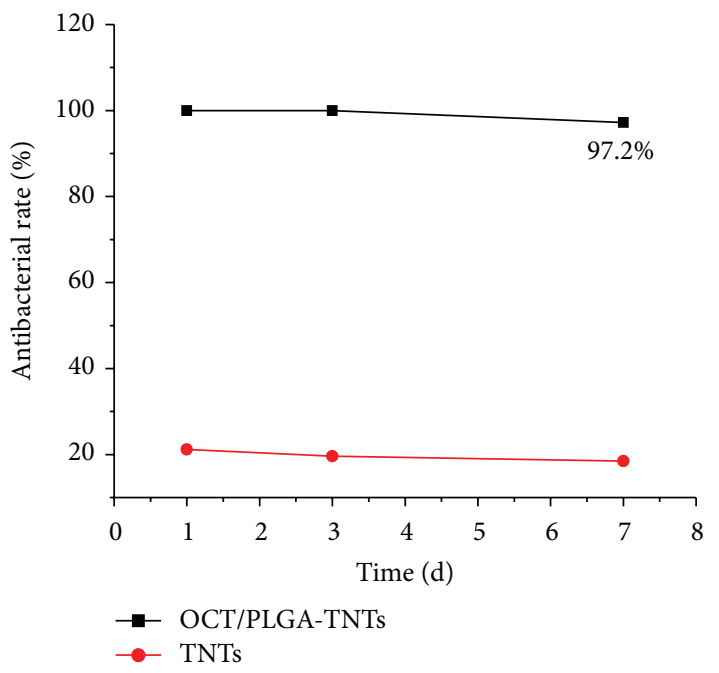

(d)

FIGURE 5: SEM images of S. aureus incubated for $6 \mathrm{~h}$ on the samples: (a) control Ti, (b) OCT/PLGA-TNTs, and (c) TNTs. (d) shows in vitro antibacterial rate profiles of the samples.

because of its excellent biocompatibility and biodegradability without interrupting osseointegration [39]. As expected, our proposed design enabled slow and sustained release for $15 \mathrm{~d}$ using the composite OCT/PLGA-TNTs. S. aureus, known for its extensive resistance to antibiotics, is the most common cause of implant infections [40] and was therefore chosen for the study. The antibacterial ability of the OCT/PLGA-TNTs decreased gradually with time and the tendency was consistent with the OCT time release profiles. A postimplantation period of $6 \mathrm{~h}$ has been identified as the "decisive period" when 


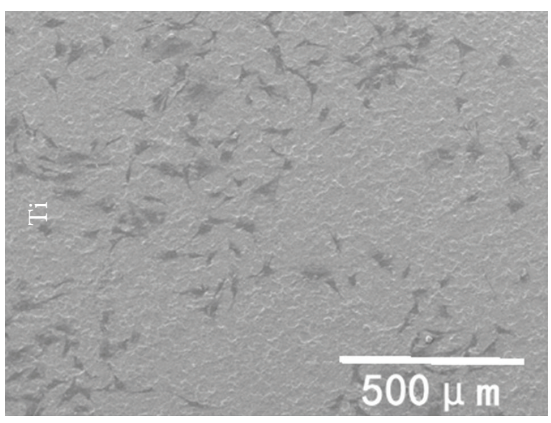

(a)

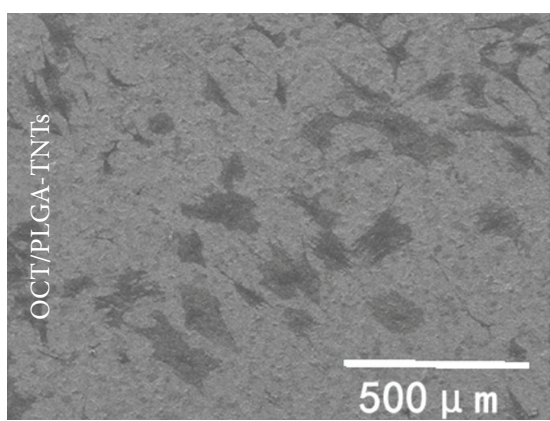

(d)

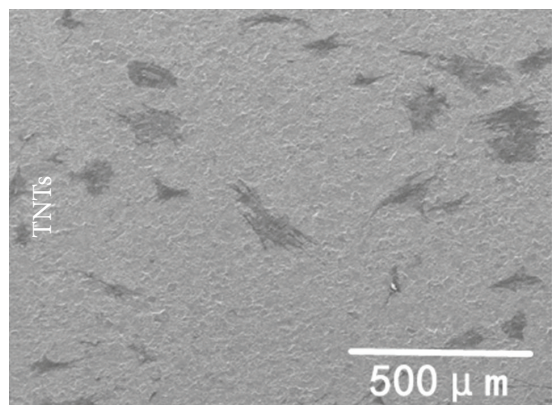

(g)

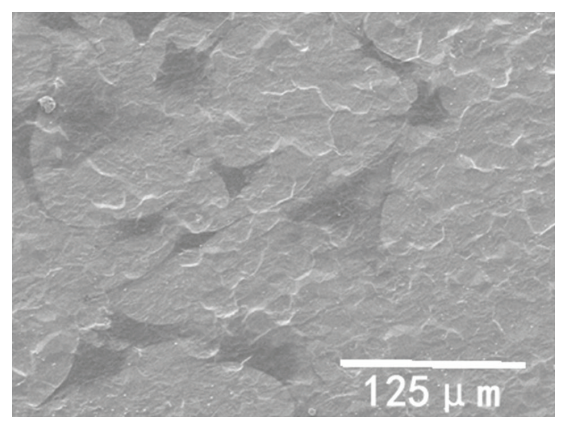

(b)

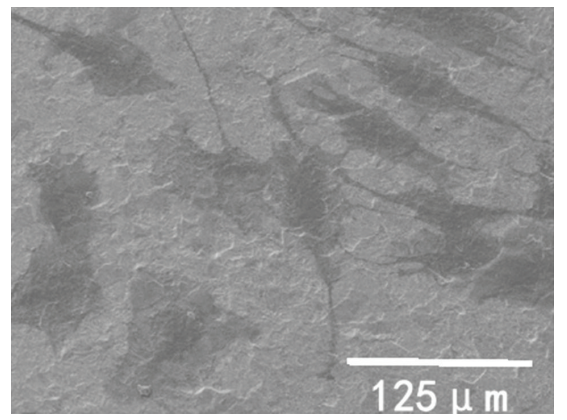

(e)

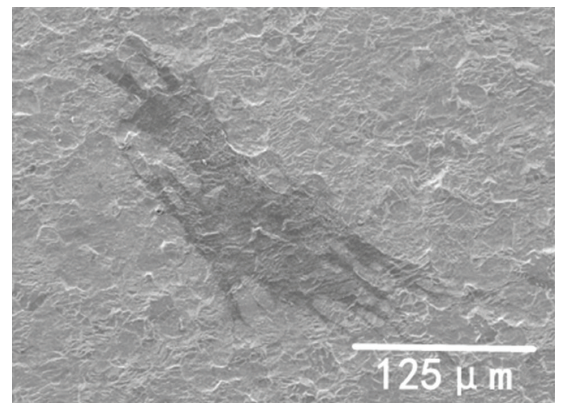

(h)

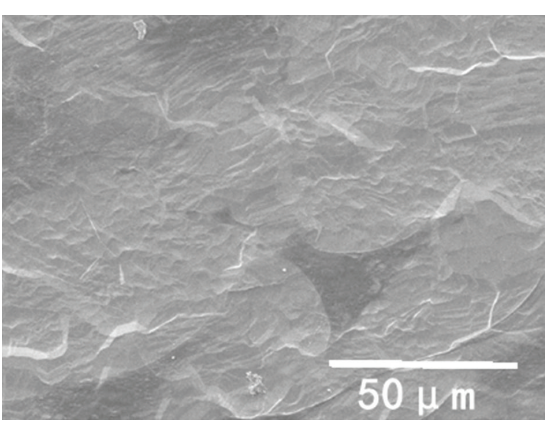

(c)

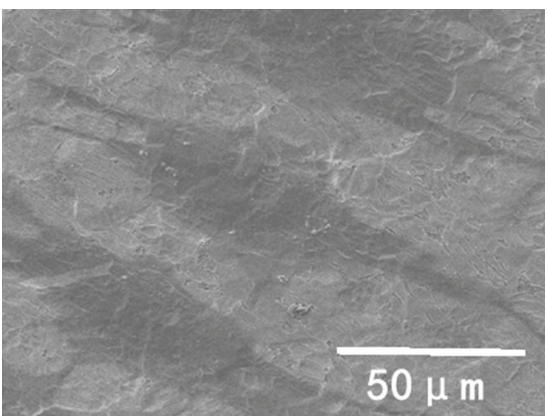

(f)

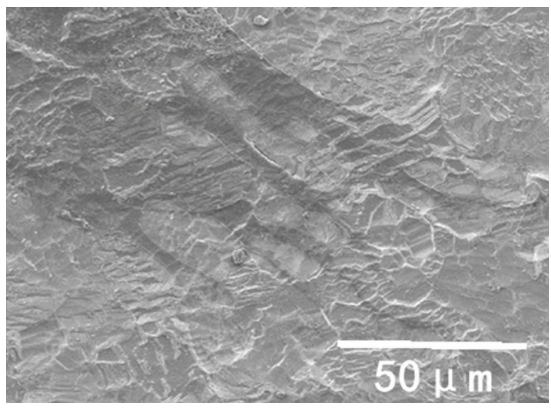

(i)

FiguRE 6: SEM pictures with three different magnifications of 200x (the first column), 800x (the second column), and 2,000x (the third column) showing the morphology of BMSCs after $2 \mathrm{~d}$ of culture on samples.

the implant is particularly susceptible to surface colonization [28]. The very few single bacterial colonies in Figure 5(b) (versus many multiple bacterial colonies in Figures 5(a) and 5 (c)) showed that the burst OCT release of $48 \%$ in the first $6 \mathrm{~h}$ could effectively inhibit bacterial adhesion on the OCT/PLGA-TNT samples, though the larger OCT release of $80 \%$ of TNTs may have resulted in less bacteria adhesion on the TNTs. After this stage, few drugs are needed to prevent further infection with the help of the host defense [41]. The antibacterial assay showed that the slow and sustained OCT release from OCT/PLGA-TNTS also exhibited a good long-term antibacterial ability within the observation period of $7 \mathrm{~d}$. The long-term antibacterial ability with slow and sustained OCT release is meaningful to preventing implant infection while avoiding potential side effects associated with OCT overdose in clinical practice. Our results also showed that TNTs without drug loading slightly inhibited the antibacterial activity. This finding is in accordance with reports that controlled titania nanotube formation through anodization and heat treatment, which decrease the contact angles of water and form crystalline $\mathrm{TiO}_{2}$, leading to a decreased bacterial adhesion on them [42].

BMSCs are multipotent stem cells that can give rise to various adult cell types, including osteoblasts [43], and most osteoblastic cells that colonize the implant surface to induce bone growth originate from BMSCs [44]. Hence, it is crucial to evaluate the behavior of BMSCs on OCT/PLGATNTs before the clinical application. Cell assay showed that OCT/PLGA-TNTs could support BMSCs adhesion and proliferation. This finding indicates that excellent antibacterial ability can be obtained from OCT at concentrations lower than the values to induce BMSC damage. This result is in accordance with previous reports $[25,45]$, in which OCT is more toxic to $S$. aureus than to mammalian cells under similar test conditions, resulting in a good biocompatibility with concomitant antibacterial activity. Furthermore, OCT/PLGATNTs promoted cell spreading and thus demonstrated excellent osteogenic activity. Although PLGA can be replaced by 

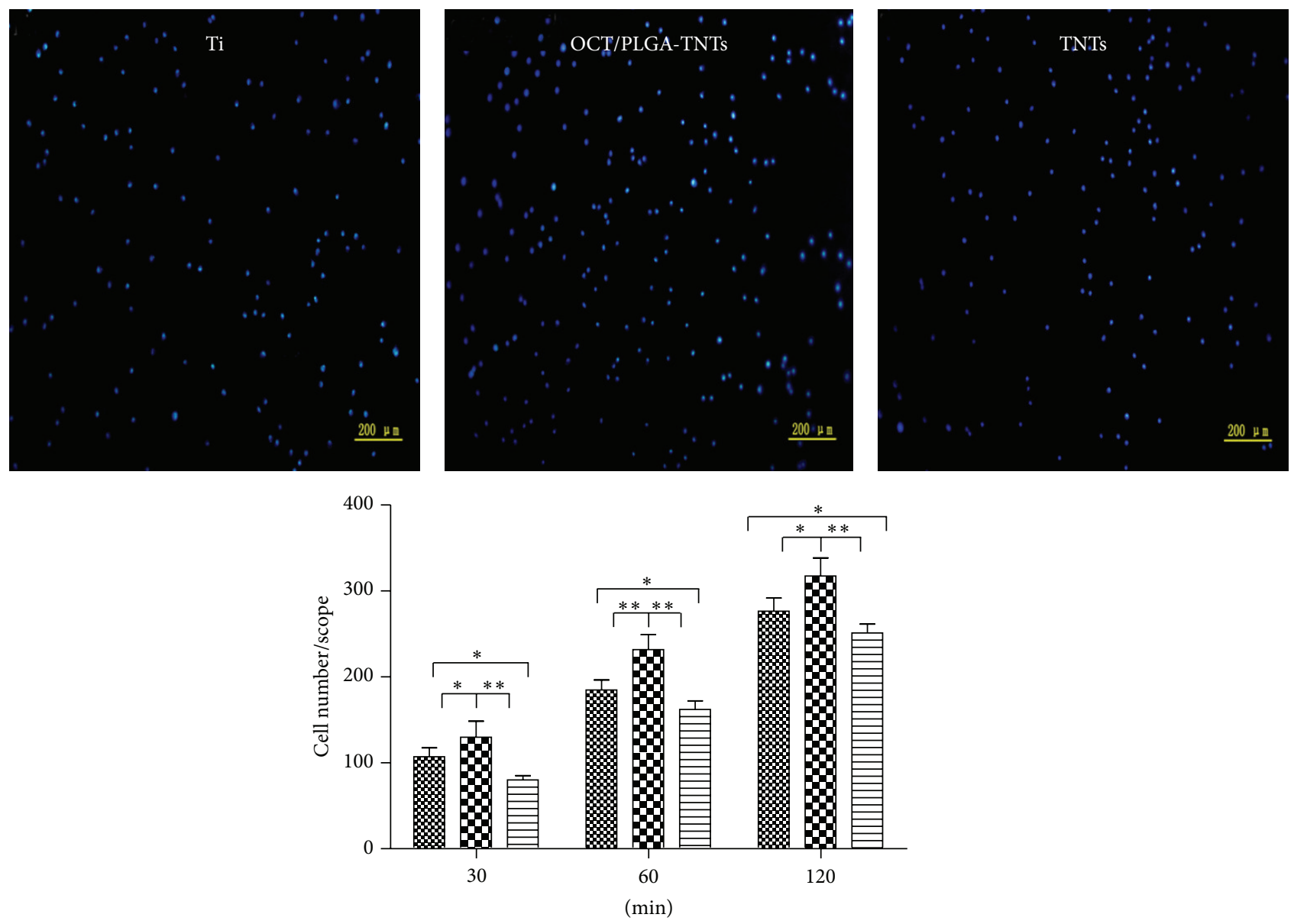

承 $\mathrm{Ti}$

OCT/PLGA-TNTs

E TNTs

FIGURE 7: Fluorescence images of initial adherent BMSCs stained with DAPI after $1 \mathrm{~h}$ and cell numbers measured by counting cells for $0.5,1$, and $2 \mathrm{~h}$ (mean $\pm \mathrm{SD}, N=5,{ }^{*} P<0.05,{ }^{* *} P<0.01$ ).

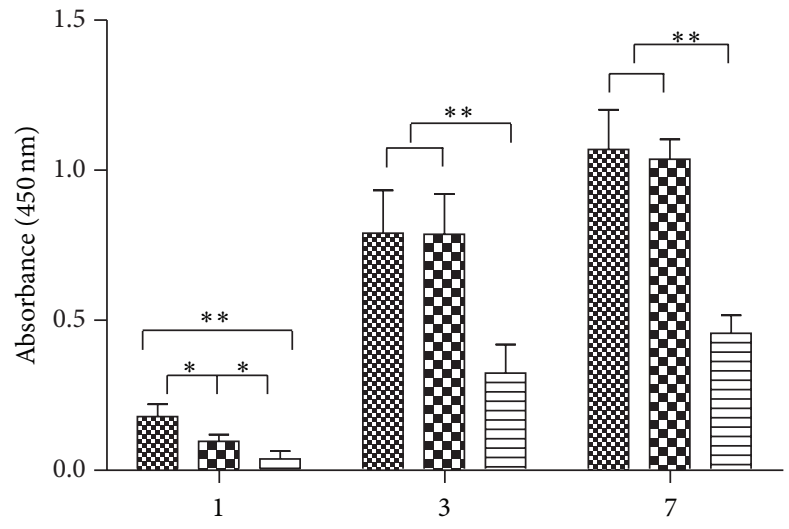

(d)

\% $\mathrm{Ti}$

OCT/PLGA-TNTs

TNTs

Figure 8: Cell proliferation measured by CCK-8 assay after culturing BMSCs on three different samples for 1,3 , and $7 \mathrm{~d}(\mathrm{mean} \pm \mathrm{SD}, N=3$, $\left.{ }^{*} P<0.05,{ }^{* *} P<0.01\right)$. 


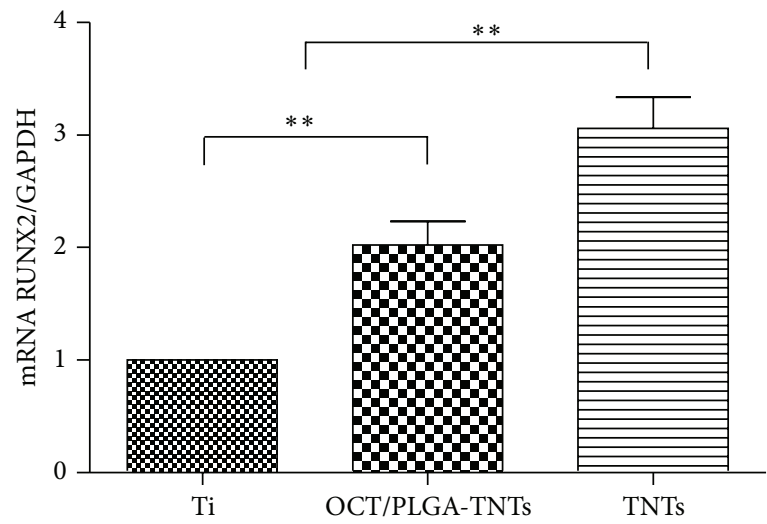

(a)

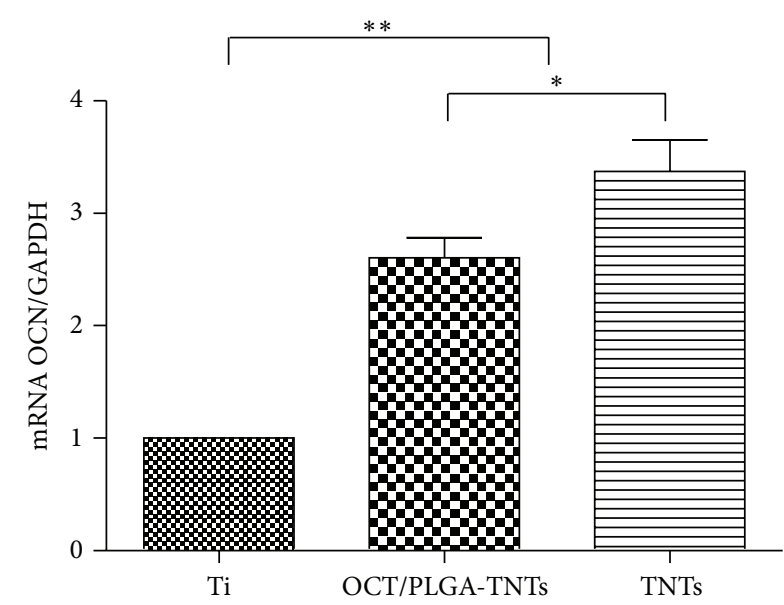

(c)

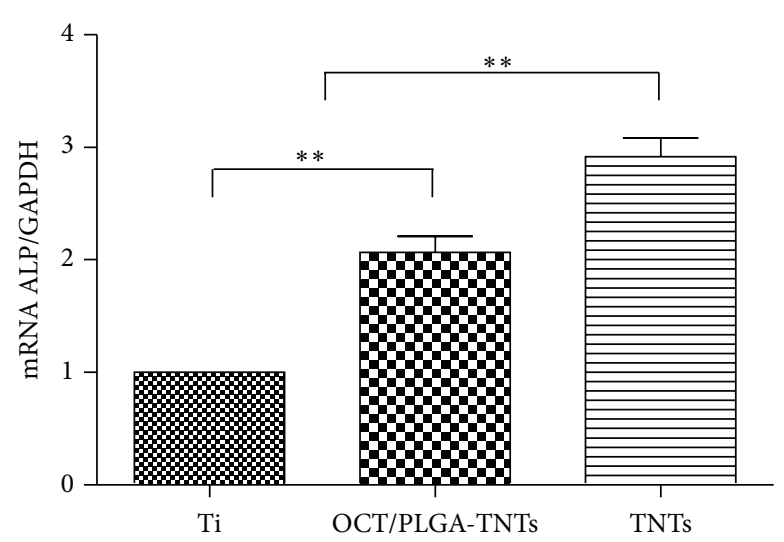

(b)

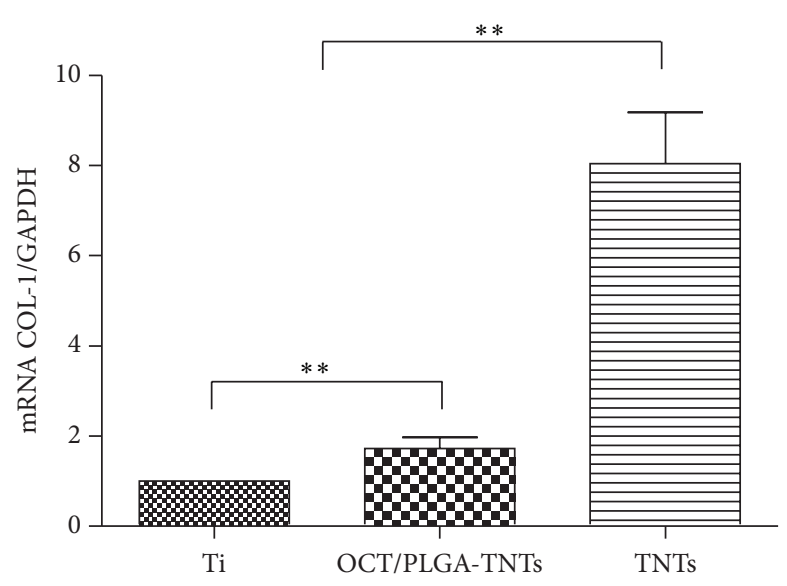

(d)

FIGURE 9: Relative expressions of (a) RUNX2, (b) ALP, (c) OCN, and (d) COL-1 by BMSCs seeding on different substrates for 2 weeks (mean $\left.\pm \mathrm{SD}, N=3,{ }^{*} P<0.05,{ }^{* *} P<0.01\right)$.

serum proteins and extracellular matrices after degradation, the initial changes (within the first $24 \mathrm{~h}$ ) in the cell shape and cytoskeletal distribution would decide the fate of stem cell differentiation [46]. In addition, a good cell distribution on the biomaterial surface is important in winning the "race for the surface" against bacteria, thereby aiding to combat infection [47]. Although TNTs alone showed the best ability to promote cell spreading and osteogenic differentiation, it obviously impaired cell adhesion and proliferation.

The technique reported in this study can be readily extended to other types of local drug-delivery applications to produce desirable biological effects. The incorporated amount and release rates can also be readily controlled by varying the drug concentration and the lactide-to-glycolide ratio.

\section{Conclusions}

In summary, OCT/PLGA was loaded into TNTs without completely filling the nanotubes using a simple solventcasting technique to obtain sustained OCT release. OCT/ PLGA-TNTs showed good antibacterial effects which could prevent postoperation complications and even late cases of infection while osteogenic activity was enhanced. Moreover, the fabrication process of OCT/PLGA-TNTs is simple, economical, and versatile. Thus, OCT/PLGA-TNTs are highly attractive for biomedical implants because of their controlled drug release, long-term antibacterial efficacy, and promotion of osseointegration.

\section{Conflict of Interests}

The authors declare that there is no conflict of interests regarding the publication of this paper.

\section{Acknowledgments}

This work is supported by the Key Specialist Facility of Fujian Province (20121589) and the Science and Technology Project of Fujian Province (2013Y0036). The authors gratefully thank the Fujian Key Laboratory of Polymer Materials for facilitating TNTs preparation. They also thank Mr. Feng Shi and Mr. Jianhe Liang for technical assistance. 


\section{References}

[1] L. Actis, L. Gaviria, T. Guda, and J. L. Ong, "Antimicrobial surfaces for craniofacial implants: state of the art," Journal of the Korean Association of Oral and Maxillofacial Surgeons, vol. 39, no. 2, pp. 43-54, 2013.

[2] J. Gallo, M. Holinka, and C. S. Moucha, "Antibacterial surface treatment for orthopaedic implants," International Journal of Molecular Sciences, vol. 15, no. 8, pp. 13849-13880, 2014.

[3] D. R. Monteiro, L. F. Gorup, A. S. Takamiya, A. C. Ruvollo-Filho, E. R. D. Camargo, and D. B. Barbosa, "The growing importance of materials that prevent microbial adhesion: antimicrobial effect of medical devices containing silver," International Journal of Antimicrobial Agents, vol. 34, no. 2, pp. 103-110, 2009.

[4] T.-F. C. Mah and G. A. O’Toole, "Mechanisms of biofilm resistance to antimicrobial agents," Trends in Microbiology, vol. 9, no. 1, pp. 34-39, 2001.

[5] K. Gulati, M. S. Aw, D. Findlay, and D. Losic, "Local drug delivery to the bone by drug-releasing implants: perspectives of nano-engineered titania nanotube arrays," Therapeutic Delivery, vol. 3, no. 7, pp. 857-873, 2012.

[6] M. Zilberman and J. J. Elsner, "Antibiotic-eluting medical devices for various applications," Journal of Controlled Release, vol. 130, no. 3, pp. 202-215, 2008.

[7] G. Gao, D. Lange, K. Hilpert et al., “The biocompatibility and biofilm resistance of implant coatings based on hydrophilic polymer brushes conjugated with antimicrobial peptides," Biomaterials, vol. 32, no. 16, pp. 3899-3909, 2011.

[8] K. S. Brammer, C. J. Frandsen, and S. Jin, “ $\mathrm{TiO}_{2}$ nanotubes for bone regeneration," Trends in Biotechnology, vol. 30, no. 6, pp. 315-322, 2012.

[9] J. Park, S. Bauer, K. von der Mark, and P. Schmuki, "Nanosize and vitality: $\mathrm{TiO}_{2}$ nanotube diameter directs cell fate," Nano Letters, vol. 7, no. 6, pp. 1686-1691, 2007.

[10] J. Park, S. Bauer, K. A. Schlegel, F. W. Neukam, K. D. von Mark, and P. Schmuki, " $\mathrm{TiO}_{2}$ nanotube surfaces: $15 \mathrm{~nm}$-an optimal length scale of surface topography for cell adhesion and differentiation," Small, vol. 5, no. 6, pp. 666-671, 2009.

[11] S. Oh, K. S. Brammer, Y. S. J. Li et al., "Stem cell fate dictated solely by altered nanotube dimension," Proceedings of the National Academy of Sciences of the United States of America, vol. 106, no. 7, pp. 2130-2135, 2009.

[12] K. S. Brammer, S. Oh, C. J. Cobb, L. M. Bjursten, H. V. D. Heyde, and S. Jin, "Improved bone-forming functionality on diametercontrolled $\mathrm{TiO}_{2}$ nanotube surface," Acta Biomaterialia, vol. 5, no. 8, pp. 3215-3223, 2009.

[13] K. C. Popat, M. Eltgroth, T. J. LaTempa, C. A. Grimes, and T. A. Desai, "Decreased Staphylococcus epidermis adhesion and increased osteoblast functionality on antibiotic-loaded titania nanotubes," Biomaterials, vol. 28, no. 32, pp. 4880-4888, 2007.

[14] G. E. Aninwene II, C. Yao, and T. J. Webster, "Enhanced osteoblast adhesion to drug-coated anodized nanotubular titanium surfaces," International Journal of Nanomedicine, vol. 3, no. 2, pp. 257-264, 2008.

[15] H. Z. Zhang, Y. Sun, A. Tian et al., "Improved antibacterial activity and biocompatibility on vancomycin-loaded $\mathrm{TiO}_{2}$ nanotubes: in vivo and in vitro studies," International Journal of Nanomedicine, vol. 8, pp. 4379-4389, 2013.

[16] P. Chennell, E. Feschet-Chassot, T. Devers, K. O. Awitor, S. Descamps, and V. Sautou, "In vitro evaluation of $\mathrm{TiO}_{2}$ nanotubes as cefuroxime carriers on orthopaedic implants for the prevention of periprosthetic joint infections," International Journal of Pharmaceutics, vol. 455, no. 1-2, pp. 298-305, 2013.

[17] W.-T. Lin, H.-L. Tan, Z.-L. Duan et al., "Inhibited bacterial biofilm formation and improved osteogenic activity on gentamicin-loaded titania nanotubes with various diameters," International Journal of Nanomedicine, vol. 9, no. 1, pp. 12151230, 2014.

[18] M. S. Aw, "Controlling drug release from titania nanotube arrays using polymer nanocarriers and biopolymer coating," Journal of Biomaterials and Nanobiotechnology, vol. 2, no. 5, pp. 477-484, 2011.

[19] K. Gulati, S. Ramakrishnan, M. S. Aw, G. J. Atkins, D. M. Findlay, and D. Losic, "Biocompatible polymer coating of titania nanotube arrays for improved drug elution and osteoblast adhesion," Acta Biomaterialia, vol. 8, no. 1, pp. 449-456, 2012.

[20] M. Kazemzadeh-Narbat, B. F. L. Lai, C. Ding, J. N. Kizhakkedathu, R. E. W. Hancock, and R. Wang, "Multilayered coating on titanium for controlled release of antimicrobial peptides for the prevention of implant-associated infections," Biomaterials, vol. 34, no. 24, pp. 5969-5977, 2013.

[21] H. Jia and L. L. Kerr, "Sustained ibuprofen release using composite poly(lactic-co-glycolic acid)/titanium dioxide nanotubes from Ti implant surface," Journal of Pharmaceutical Sciences, vol. 102, no. 7, pp. 2341-2348, 2013.

[22] R. Johnson, "Antibiotics: resistance is restored," Nature Chemistry, vol. 6, no. 9, pp. 754-755, 2014.

[23] N.-O. Hübner, J. Siebert, and A. Kramer, "Octenidine dihydrochloride, a modern antiseptic for skin, mucous membranes and wounds," Skin Pharmacology and Physiology, vol. 23, no. 5, pp. 244-258, 2010.

[24] T. Koburger, N.-O. Hübner, M. Braun, J. Siebert, and A. Kramer, "Standardized comparison of antiseptic efficacy of triclosan, PVP-iodine, octenidine dihydrochloride, polyhexanide and chlorhexidine digluconate," Journal of Antimicrobial Chemotherapy, vol. 65, no. 8, pp. 1712-1719, 2010.

[25] G. Baier, A. Cavallaro, K. Friedemann et al., "Enzymatic degradation of poly(l-lactide) nanoparticles followed by the release of octenidine and their bactericidal effects," Nanomedicine: Nanotechnology, Biology, and Medicine, vol. 10, no. 1, pp. 131-139, 2014.

[26] S. A. Baskaran, A. Upadhyay, I. Upadhyaya, V. Bhattaram, and K. Venkitanarayanan, "Efficacy of octenidine hydrochloride for reducing Escherichia coli O157: H7, Salmonella spp., and Listeria monocytogenes on cattle hides," Applied and Environmental Microbiology, vol. 78, no. 12, pp. 4538-4541, 2012.

[27] S. Weckbach, A. Möricke, H. Braunwarth, P. Goroncy-Bermes, M. Bischoff, and F. Gebhard, "Octenidine in combination with polymethylmethacrylate: a new option for preventing infection?" Archives of Orthopaedic and Trauma Surgery, vol. 132, no. 1, pp. 15-20, 2012.

[28] K. G. Neoh, X. Hu, D. Zheng, and E. T. Kang, "Balancing osteoblast functions and bacterial adhesion on functionalized titanium surfaces," Biomaterials, vol. 33, no. 10, pp. 2813-2822, 2012.

[29] F. Grinnell and M. K. Feld, "Fibronectin adsorption on hydrophilic and hydrophobic surfaces detected by antibody binding and analyzed during cell adhesion in serum-containing medium," The Journal of Biological Chemistry, vol. 257, no. 9, pp. 4888-4893, 1982.

[30] K. Webb, V. Hlady, and P. A. Tresco, "Relative importance of surface wettability and charged functional groups on NIH 
3T3 fibroblast attachment, spreading, and cytoskeletal organization," Journal of Biomedical Materials Research, vol. 41, pp. 422-430, 1998.

[31] A. M. G. Borges, L. O. Benetoli, M. A. Licínio et al., "Polymer films with surfaces unmodified and modified by non-thermal plasma as new substrates for cell adhesion," Materials Science and Engineering C, vol. 33, no. 3, pp. 1315-1324, 2013.

[32] Y. Wang, C. Wen, P. Hodgson, and Y. Li, "Biocompatibility of $\mathrm{TiO}_{2}$ nanotubes with different topographies," Journal of Biomedical Materials Research-Part A, vol. 102, no. 3, pp. 743-751, 2014.

[33] X. Liu, Q. Feng, A. Bachhuka, and K. Vasilev, "Surface chemical functionalities affect the behavior of human adipose-derived stem cells in vitro," Applied Surface Science, vol. 270, pp. 473479, 2013.

[34] G. S. Stein, J. B. Lian, and T. A. Owen, "Relationship of cell growth to the regulation of tissue-specific gene expression during osteoblast differentiation," The FASEB Journal, vol. 4, no. 13, pp. 3111-3123, 1990.

[35] I. Wall, N. Donos, K. Carlqvist, F. Jones, and P. Brett, "Modified titanium surfaces promote accelerated osteogenic differentiation of mesenchymal stromal cells in vitro," Bone, vol. 45, no. 1, pp. 17-26, 2009.

[36] E. A. Cavalcanti-Adam, D. Aydin, V. C. Hirschfeld-Warneken, and J. P. Spatz, "Cell adhesion and response to synthetic nanopatterned environments by steering receptor clustering and spatial location," HFSP Journal, vol. 2, no. 5, pp. 276-285, 2008.

[37] R. McBeath, D. M. Pirone, C. M. Nelson, K. Bhadriraju, and C. S. Chen, "Cell shape, cytoskeletal tension, and RhoA regulate stem cell lineage commitment," Developmental Cell, vol. 6, no. 4, pp. 483-495, 2004.

[38] K. A. Kilian, B. Bugarija, B. T. Lahn, and M. Mrksich, "Geometric cues for directing the differentiation of mesenchymal stem cells," Proceedings of the National Academy of Sciences of the United States of America, vol. 107, no. 11, pp. 4872-4877, 2010.

[39] S. Y. Lee, J. Y. Koak, S. J. Heo, S. K. Kim, S. J. Lee, and S. Y. Nam, "Osseointegration of anodized titanium implants coated with poly(lactide-co-glycolide)/basic fibroblast growth factor by electrospray," The International Journal of Oral \& Maxillofacial Implants, vol. 25, no. 2, pp. 315-320, 2010.

[40] D. Campoccia, L. Montanaro, and C. R. Arciola, "The significance of infection related to orthopedic devices and issues of antibiotic resistance," Biomaterials, vol. 27, no. 11, pp. 2331-2339, 2006.

[41] L. Zhao, P. K. Chu, Y. Zhang, and Z. Wu, "Antibacterial coatings on titanium implants," Journal of Biomedical Materials Research. Part B Applied Biomaterials, vol. 91, no. 1, pp. 470-480, 2009.

[42] B. Ercan, E. Taylor, E. Alpaslan, and T. J. Webster, "Diameter of titanium nanotubes influences anti-bacterial efficacy," Nanotechnology, vol. 22, no. 29, Article ID 295102, 2011.

[43] C. Vater, P. Kasten, and M. Stiehler, "Culture media for the differentiation of mesenchymal stromal cells," Acta Biomaterialia, vol. 7, no. 2, pp. 463-477, 2011.

[44] R. Tasso, F. Fais, D. Reverberi, F. Tortelli, and R. Cancedda, "The recruitment of two consecutive and different waves of host stem/progenitor cells during the development of tissueengineered bone in a murine model," Biomaterials, vol. 31, no. 8, pp. 2121-2129, 2010.
[45] G. Müller and A. Kramer, "Biocompatibility index of antiseptic agents by parallel assessment of antimicrobial activity and cellular cytotoxicity," The Journal of Antimicrobial Chemotherapy, vol. 61, no. 6, pp. 1281-1287, 2008.

[46] K. Kolind, K. W. Leong, F. Besenbacher, and M. Foss, "Guidance of stem cell fate on 2D patterned surfaces," Biomaterials, vol. 33, no. 28, pp. 6626-6633, 2012.

[47] A. Gao, R. Hang, X. Huang et al., "The effects of titania nanotubes with embedded silver oxide nanoparticles on bacteria and osteoblasts," Biomaterials, vol. 35, no. 13, pp. 4223-4235, 2014. 

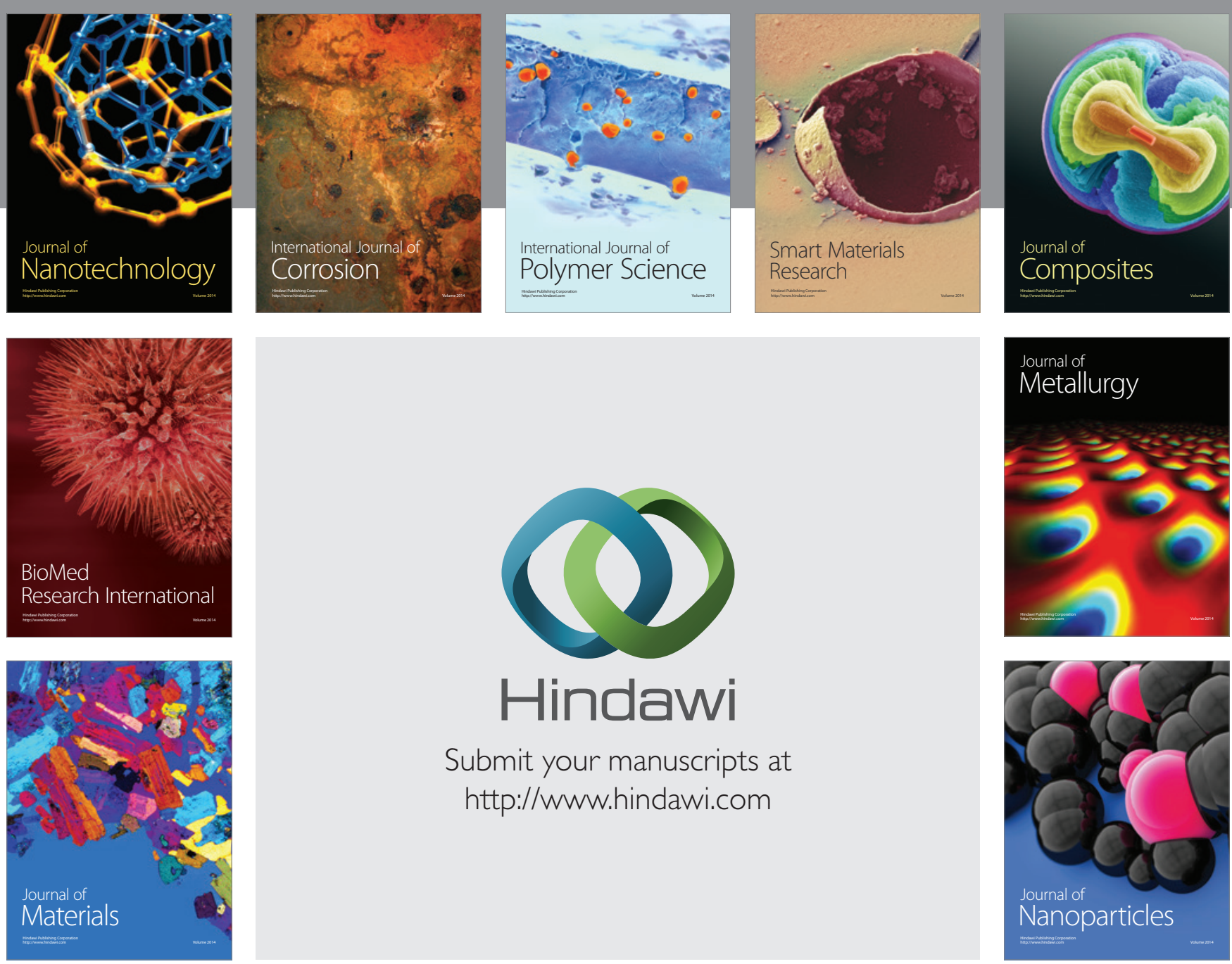

Submit your manuscripts at http://www.hindawi.com
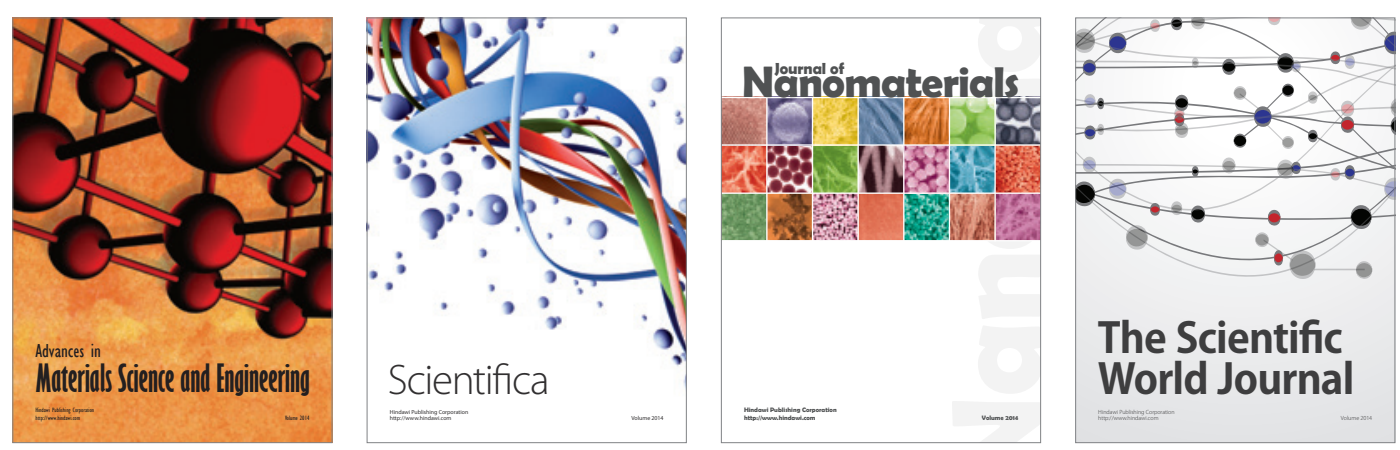

\section{The Scientific World Journal}
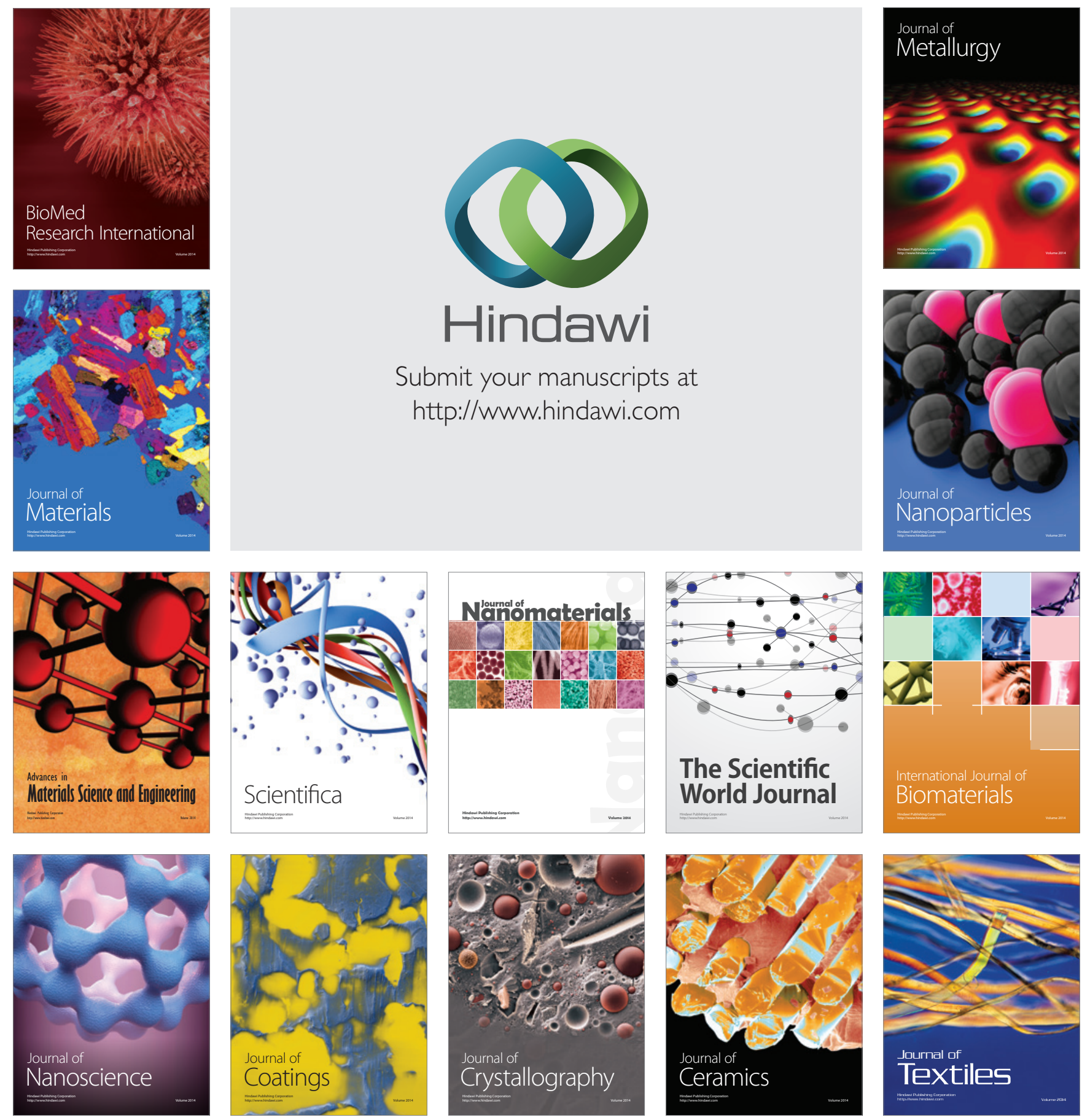